\title{
Effect of rituximab in MCNS: a role for IL-13 suppression?
}

\section{Ji Eun Kim, Se Jin Park, Tae-Sun Ha and Jae II Shin}

We read with great interest the article by Aditi Sinha and Arvind Bagga (Rituximab therapy in nephrotic syndrome: implications for patients' management. Nat. Rev. Nephrol. 9, 154-169; 2013). ${ }^{1}$ These authors reported the beneficial effects of rituximab treatment in patients with nephrotic syndrome and speculated that rituximab might cause apoptosis via complement-dependent cytotoxicity and antibody-dependent cytotoxicity, leading to rapid depletion of B cells. ${ }^{1}$ Furthermore, rituximab is reported to induce T-regulatory and B-regulatory cell populations, restore immune tolerance, and attenuate the downregulation of sphingomyelinase-like phosphodiesterase $3 \mathrm{~b}$ in podocytes. ${ }^{1}$

We would like to add another possible mechanism that may explain the beneficial effect of rituximab on minimal-change nephrotic syndrome (MCNS). Although rare, some reports have shown that Th2 cytokines, such as interleukin (IL)-13, may play an important role in the pathogenesis of MCNS. ${ }^{2-4}$ Yap et al. reported that $\mathrm{CD} 4^{+}$ and $\mathrm{CD}^{+}$IL-13 mRNA expression was increased in patients with nephrotic relapse compared with those in remission, healthy individuals, and patient controls (children with viral infections, but without idiopathic nephrotic syndrome; $P<0.008$ ) and that this finding was related to an increase in expression of cytoplasmic IL-13 in phorbol myristate acetate/ionomycin-activated $\mathrm{CD}^{+}$cells $(6.66 \pm 3.39 \%)$ in patients with nephrotic relapse compared with expression in patients in the remission phase $(2.59 \pm 1.35 \%, P<0.0001) .{ }^{2}$ Yap et al. speculated that IL-13 may act on monocytes to produce vascular permeability factors involved in the pathogenesis of proteinuria in patients with relapse of nephrotic syndrome. ${ }^{2}$ Cheung et al. also showed that the percentage of $\mathrm{CD}^{+}$IL-13-producing cells was significantly increased in children with nephrotic relapse, and correlated with serum IgE levels during the active phase of the disease $(r=0.90, P<0.001){ }^{3}$

In addition, Lai et al. have demonstrated that IL-13-transfected rats $(n=41)$ show a minimal-change-like nephropathy (characterized by increased proteinuria, hypoalbuminaemia and hypercholesterolaemia) compared with control rats $(n=17){ }^{4}$ In their study, the glomeruli of rats transfected with IL-13 showed no significant histologic changes, but electron microscopy findings showed that up to $80 \%$ of podocyte foot processes showed fusion and that glomerular gene expression of $B 7-1, I L-4 R \alpha$ and $I L-13 R \alpha 2$ were upregulated, whereas those of nephrin, podocin and dystroglycan were downregulated. ${ }^{4}$

Although not in the setting of MCNS, another study has shown that rituximab could reduce levels of IL-13 expressing T cells. ${ }^{5}$ Simon et al. showed that the numbers of cytokine-expressing $\mathrm{CD}^{+}$ and $\mathrm{CD} 8^{+} \mathrm{T}$ cells were reduced with a relative decrease in the mRNA expression of IL-5 (mean 53\%) and IL-13 (mean 83\%) after rituximab therapy in patients with moderate to severe atopic dermatitis not responding to topical corticosteroid and/or calcineurin inhibitor therapy. ${ }^{5}$

So there is a possibility that rituximab might be beneficial in MCNS by suppression of IL-13, which could be involved in the development of MCNS, in addition to depletion of B cells. However, further studies are necessary to evaluate serial changes in levels of IL-13 after rituximab treatment in MCNS and to elucidate the exact role of IL-13 in the pathogenesis of MCNS.

Yonsei-Ro 50, Seodaemun-Ku, 120-752, C.P.O. Box 8044, Department of Pediatrics, Yonsei University College of Medicine, Severance Children's Hospital, Seoul, Korea (J. E. Kim, J. I. Shin). Department of Pediatrics, Ajou University School of Medicine, San 5, Woncheondong, Yeongtong-gu, Suwon 443-721, Korea (S. J. Park). Department of Pediatrics, College of Medicine, Chungbuk National University, 410

Sung Bong-Ro, Heungduk-gu, Cheongju

361-240, South Korea (T.-S. Ha).

Correspondence to: J. I. Shin

shinji@yuhs.ac

Acknowledgements

The authors' work is supported by the Basic Science Research Program through the National Research Foundation of Korea funded by the Ministry of Education, Science and Technology (2011-0013789).

\section{Competing interests}

The authors declare no competing interests.

1. Sinha, A. \& Bagga, A. Rituximab therapy in nephrotic syndrome: implications for patients' management. Nat. Rev. Nephrol. 9, 154-169 (2013).

2. Yap, H. K. et al. Th1 and Th2 cytokine mRNA profiles in childhood nephrotic syndrome: evidence for increased IL-13 mRNA expression in relapse. J. Am. Soc. Nephrol. 10, 529-537 (1999).

3. Cheung, W., Wei, C. L., Seah, C. C., Jordan, S. C. \& Yap, H. K. Atopy, serum IgE, and interleukin-13 in steroid-responsive nephrotic syndrome. Pediatr. Nephrol. 19, 627-632 (2004).

4. Lai, K. W. et al. Overexpression of interleukin-13 induces minimal-change-like nephropathy in rats. J. Am. Soc. Nephrol. 18, 1476-1485 (2007).

5. Simon, D. \& Simon, H. U. New drug targets in atopic dermatitis. Chem. Immunol. Allergy 96 , 126-131 (2012). 